\title{
FÊNOMENO DE RAYNAUD GRAVE ASSOCIADO A TERAPIA COM INTERFERON-BETA PARA ESCLEROSE MÚLTIPLA
}

\author{
RELATO DE CASO
}

\author{
BORIS AFONSO CRUZ*, EUSTÁQUIO DE QUEIROZ**, SIMONE VILELA NUNES***, \\ ACHILES CRUZ FILHO****, GILBERTO BELISARIO CAMPOS*****, \\ ERNESTO LENTZ DE CARVALHO MONTEIRO******, HUMBERTO CRIVELLARI*******
}

\begin{abstract}
RESUMO - Interferon-B (IFN-beta) é usado no tratamento de esclerose múltipla (EM). Descrevemos o caso de uma mulher com EM que apresentou fenômeno de Raynaud grave, livedo reticular e necrose digital duas semanas após tratamento com IFN-beta. Os sintomas melhoraram após suspensão do IFN-beta e início de anticoagulação associada a ciclofosfamida e corticóide. Fenômeno de Raynaud é um efeito colateral provável da terapia com IFN-beta para EM.
\end{abstract}

PALAVRAS-CHAVE: esclerose múltipla, interferon- $\beta$, fenômeno de Raynaud

\section{Severe Raynaud's phenomenon associated with interferon-beta therapy for multiple sclerosis: case report}

ABSTRACT - Interferon- $\beta$ (IFN-beta) is administered for treatment of multiple sclerosis (MS). We report on a woman with MS who presented with severe Raynaud's phenomenon, livedo-reticularis and digital necrosis two weeks after beginning therapy with IFN-beta. Symptoms improved after the IFN-beta was discontinued and anticoagulation associated with cyclophosphamide and corticoid were introduced. Raynaud's phenomenon is probably a side effect of IFN-beta therapy for multiple sclerosis.

KEY WORDS: multiple sclerosis, interferon- $\beta$, Raynaud's phenomenon

Esclerose múltipla (EM) é uma patologia neurológica crônica caracterizada por inflamação da substância branca, desmielinização e esclerose glial. Clinicamente se traduz por disfunção neurológica progressiva, na maioria dos pacientes em um curso de ataques de exacerbação e remissão, chamando atenção a carência de tratamento específico ${ }^{1}$. Interferon- $\beta$ (IFN- $\beta$ ) mostrou-se eficaz em reduzir a frequência e severidade dos ataques de EM, motivo pelo qual vem sendo usado na prática clínica. Seus principais efeitos colaterais são sintomas gripais, reações cutâneas no sítio de aplicação e elevação de enzimas hepáticas ${ }^{2,3}$. Neste estudo, descrevemos uma paciente com diagnóstico de EM que duas semanas após o início do tratamento com IFN-beta apresentou fenômeno de Raynaud exuberante e necrose digital, um provável efeito colateral a ser relatado na literatura.

Serviço de Neurologia do Hospital Governador Israel Pinheiro (HGIP), Belo Horizonte, Brasil: *Residente de Reumatologia do HGIP; **Residente de Clínica Médica do HGIP; ***Residente de Neurologia do HGIP; ****Doutor e Livre-Docente em Reumatologia da Faculdade de Medicina da Universidade Federal de Minas Gerais (UFMG); *****Preceptor do Serviço de Neurologia do HGIP, Professor Emérito de Neurologia da FMUFMG; *******Professor Adjunto IV de Cirurgia Cardiovascular da FM-UFMG; *******Chefe do Serviço de Neurologia do HGIP. Aceite: 10-março-2000.

Dr. Boris Afonso Cruz - Rua do Mosteiro 105/301 - 30380-780 Belo Horizonte MG - Brasil. E-mail: borisc@net.em.com.br 


\section{CASO}

Uma mulher de 58 anos com diagnóstico de EM foi referida para avaliação reumatológica por um dos autores (ACF) por fenômeno de Raynaud e livedo reticular no membro superior esquerdo, com suspeita diagnóstica de vasculite. A paciente apresentava história de paraparesia e parestesias esporádicas de membros inferiores com remissão espontânea, iniciados há 12 anos. Passou a apresentar nos últimos 3 anos crises mais frequentes, surgindo comprometimento de membros superiores, distúrbios visuais, vertigem, parestesia na face e sensação de "choque" na coluna vertebral. Combinava estes sintomas em crises distintas, satisfazendo os critérios de Poser et al. para EM clinicamente definida ${ }^{4}$. Avaliação radiológica por ressonância nuclear magnética corroborava o diagnóstico, evidenciando em T-2 várias lesões em placa acometendo substância branca difusamente, incluindo lesões periventriculares e no tronco cerebral. Apesar do tratamento com azatioprina (100 mg/dia), a paciente evoluía com sintomas frequentes e déficit cognitivo progressivo. Tais crises eram abordadas com "pulsoterapias" de metil-prednisolona seguidas de prednisona oral. Pela falência do tratamento acima descrito, foi instituído interferon beta-1b (Betaferon ${ }^{\circledR}$, Schering, Brasil) na dose de 8 milhões de unidades por via subcutânea em dias alternados. Duas semanas após o início do tratamento com IFN- $\beta$, surgiu cianose exacerbada pelo frio em dedos da mão esquerda, seguindo-se livedo reticular no antebraço esquerdo e dor intensa no IV dedo da mão esquerda. Estava em uso de prednisona ( $40 \mathrm{mg} / \mathrm{dia}$ ), cloridrato de imipramina ( $20 \mathrm{mg} / \mathrm{dia}$ ) e baclofeno ( $10 \mathrm{mg}, 3$ a 4 vezes por dia), todos estes iniciados antes do IFN- $\beta$. Ao exame clínico, apresentava livedo reticular nítido na superfície dorsal do antebraço esquerdo e cianose importante dos dedos da mão esquerda, já se percebendo necrose isquêmica com limites imprecisos na polpa digital do IV dedo da mão esquerda (Fig 1). Eram evidentes déficit de memória importante, força muscular diminuída no membro superior esquerdo (3/5), paraparesia espástica com nível sensitivo em T7 e marcha com base alargada, necessitando de "andador" para locomover-se. O restante do exame era normal. O IFN- $\beta$ foi prontamente suspenso, diante da suspeita de que os fenômenos vasculares poderiam constituir efeito colateral desta medicação, enquanto se realizavam biópsia de pele e estudo laboratorial.

Do ponto de vista de exames complementares a paciente apresentava hemograma sem alteração, hemossedimentação de $29 \mathrm{~mm} /$ hora, proteína C reativa, fator reumatóide, FAN, ANCA, pesquisa de crioglobulinemia, pesquisa de trombofilia (proteína C, proteína S, anti-trombina III, fator V de Leyden) e anticorpos anti-fosfolípides (anticoagulante lúpico e anti-cardiolipina $\operatorname{IgG}$ e $\operatorname{IgM}$ ) normais ou negativos. Foi realizada biópsia na região do livedo reticular que evidenciou discreto infiltrado linfocitário perivascular (Fig 2).

Duas semanas após a suspensão do IFN- $\beta$, permaneciam os sintomas cutâneos e dor no IV dedo da mão esquerda. Ocorriam ainda episódios de vertigem e amaurose fugaz. Optou-se pela anticoagulação plena com varfarina, nos primeiros 5 dias associada a heparina, além da introdução de "pulsoterapia" combinando e ciclofosfamida ( $1 \mathrm{~g})$ e metilprednisolona $(1 \mathrm{~g})$, seguida de prednisona oral na dose de $60 \mathrm{mg} / \mathrm{dia}$. Já nos primeiros dias a paciente evoluiu com resolução da dor, definição da necrose na polpa digital do IV dedo da mão esquerda e melhora importante da cianose. Do ponto de vista neurológico, persistiam os déficits motores no membro superior esquerdo e membros inferiores, porém ocorreu melhora importante do déficit de memória e da vertigem, além de resolução da amaurose.

Após seguimento de 8 semanas, a paciente encontrava-se anticoagulada, em uso de ciclofosfamida em doses endovenosas mensais de $1 \mathrm{~g}$, associada a prednisona oral $40 \mathrm{mg} /$ dia, sem novos sintomas vasculares ou neurológicos.

\section{DISCUSSÃO}

Interferons são citocinas produzidas em resposta a certos vírus, bactérias e outros antígenos. Têm função antiviral, antiproliferativa e imunomoduladora, sendo três os tipos descritos: alfa, beta e gama ${ }^{5}$. Em dois grandes estudos sobre terapia com IFN- $\beta$ para EM poucos efeitos colaterais foram descritos, sendo que nenhum paciente desenvolveu fenômenos autoimunes ou isquêmicos ${ }^{2,3}$. Já a terapia com interferon- $\alpha$ (IFN- $\alpha$ ) vem sendo associada a exacerbação de doenças autoimunes ${ }^{6,7}$ e fenômeno de Raynaud com necrose digital ${ }^{8}$. Especula-se que tal efeito ocorra devido à atividade imunomoduladora dos interferons, através de sua participação na expressão de moléculas HLA classe II, ativação de macrófagos e linfócitos T citotóxicos e influência na produção de anticorpos ${ }^{5}$. Como atuam sobre os mesmos receptores, é possível que IFN- $\alpha$ e IFN- $\beta$ sejam capazes de provocar os mesmos efeitos por mecanismos comuns. No entanto, permanece incerto porque tais efeitos são mais frequentes com IFN- $\alpha$, já que os esquemas de posologia são similares ${ }^{3}$. Esta diferença pode estar relacionada às doenças a serem tratadas e não à terapêutica propriamente dita. Por exemplo: hepatite $\mathrm{C}$, para qual se utiliza IFN- $\alpha$, pode estar associada a produção de anticorpos e 


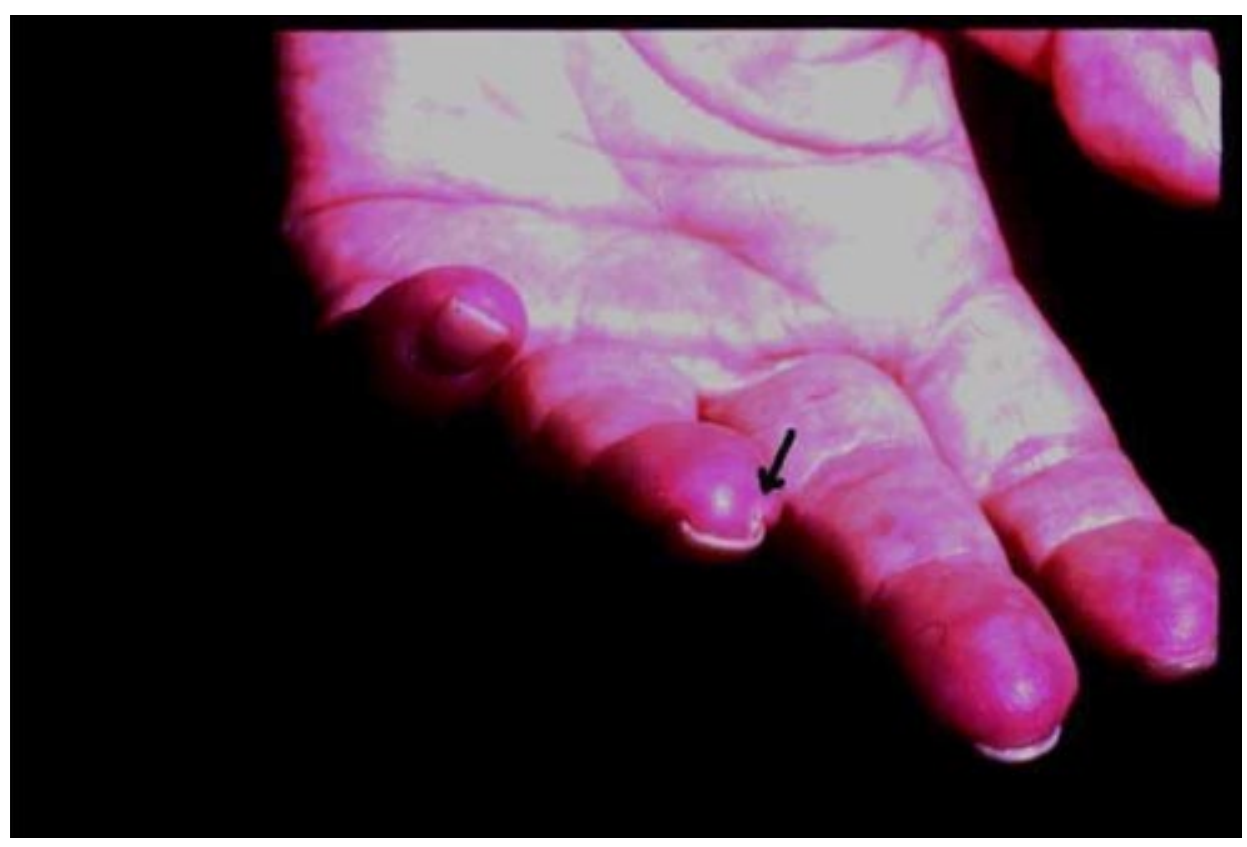

Fig 1. Fenômeno de Raynaud e necrose em polpa digital do terceiro dedo da mão esquerda (seta)

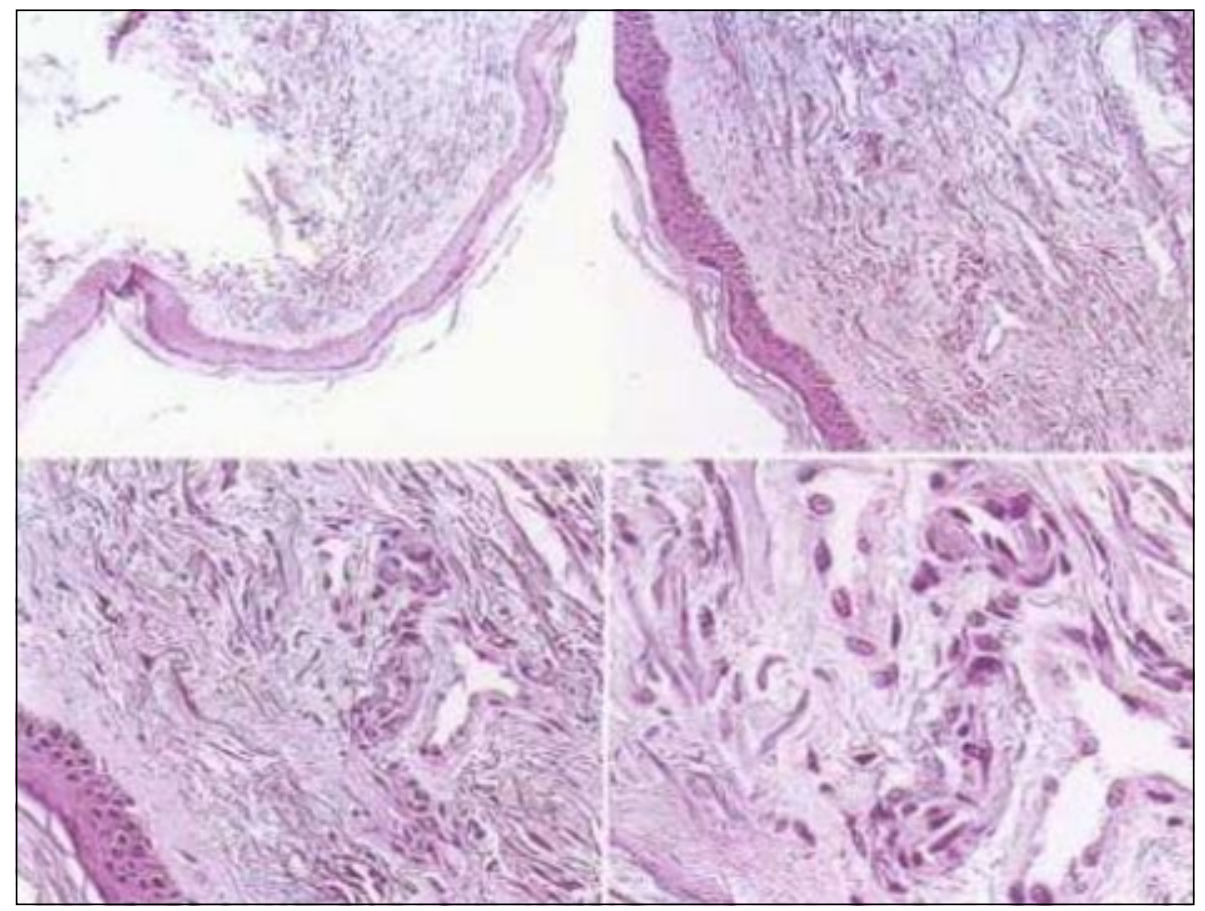

Fig 2. A biópsia de pele evidencia epiderme hipotrófica, retificada, com discreta ceratose. A derme tem degeneração basofílica e apresenta exsudato mononuclear em torno de vasos de pequeno calibre. A imunofluorescência foi negativa para depósitos de $\operatorname{IgA,~} \operatorname{IgG}$, IgM e C3. 
desenvolvimento de fenômenos autoimunes incluindo vasculopatias ${ }^{9}$, ao passo que a associação de EM e desordens inflamatórias autoimunes é infrequente ${ }^{10}$.

Em revisão da literatura médica encontram-se relatos isolados da associação de IFN- $\beta$ e manifestações autoimunes, notadamente artrite reumatóide ${ }^{7,11}$. No que diz respeito a fenômenos vasculares, Linden descreve um caso semelhante ao aqui apresentado, em que uma paciente com EM apresentou fenômeno de Raynaud associado a necrose digital após terapia com IFN- $\beta$. Os sintomas evanesceram após suspensão do IFN- $\beta$ e anticoagulação ${ }^{12}$.

É necessário ainda comentar que as crises de vertigem e amaurose fugaz também se intensificaram com IFN- $\beta$ e se resolveram após suspensão deste e início de anticoagulação associada à terapêutica imunossupressora. Apesar de tais manifestações fazerem parte do quadro clínico de EM, a relação acima apresentada aponta a possibilidade de componente vascular também na exacerbação destes sintomas. Não foi realizada avaliação mais apurada de tais queixas, especialmente do ponto de vista de exames complementares, o que impede a caracterização de uma relação de causa e efeito entre IFN- $\beta$ e estes sintomas. Entretanto, é descrita a associação de IFN- $\alpha$ e outras manifestações isquêmicas além do fenômeno de Raynaud como isquemia coronária ${ }^{13}$, colite isquêmica ${ }^{14}$, neuropatia óptica anterior isquêmica ${ }^{15}$ e vasoespasmo na retina levando a oclusão de capilares retinianos ${ }^{16}$. Pela similaridade dos mecanismos de ação de IFN- $\beta$ e IFN- $\alpha^{3}$, estes dados sugerem, ao menos teoricamente, que IFN- $\beta$ poderia ser capaz de desencadear fenômenos isquêmicos.

A relação cronológica nítida entre o fenômeno de Raynaud e a terapia com IFN- $\beta$ associada aos dados da literatura médica aqui apresentados, sugerem que IFN- $\beta$ teria participação na gênese destes sintomas vasculares, possivelmente por mecanismo imunomodulador. Pelo crescente uso de IFN- $\beta$ para o tratamento de EM, chamamos atenção para a possibilidade de manifestações vasculares como possível intercorrência durante o tratamento, além de recomendar monitorização clínica cuidadosa destes pacientes, no sentido de se prevenir complicações isquêmicas.

\section{REFERÊNCIAS}

1. Sadik AS, Miller JR. Multiple sclerosis. In Rowland LP. Merritt's textbook of Neurology. 9Ed. New York: Wiliams and Wilkins, 1995;804-825.

2. Jacobs LD, Cookfair DL, Rudick RA, et al. Intramuscular interferon beta-1a for disease progression in relapsing multiple sclerosisAnn Neurol 1996;39:285-294.

3. The IFNB Multiple Sclerosis Study Group and The University of British Columbia MS/MRI Analysis Group. Interferon beta-1b in the treatment of multiple sclerosis: final outcome of the randomized controlled trial. Neurology 1995;45:1277-1285.

4. Poser CM, Patty DW, Scheinberg L, et al. New diagnostic criteria for multiple sclerosis: guidelines for research protocols. Ann Neurol 1983;13:227-231.

5. Durum SK, Oppenheim JJ. Proinflamatory cytokines and immunity. In Paul WE (ed). Fundamental immunology. New York: Raven Press, 1993:815-819.

6. Conlon KC, Urba WJ, Smith JW, et al. Exacerbation of symptoms of autoimmune disease in patients receiving alphainterferon therapy. Cancer 1990;65:2237-2242.

7. Levesque MC, Ward FE, Douglas RJ, Weinberg JB. Interferon- $\beta 1 \mathrm{~A}$ induced polyarthritis in a patient with the HLADRB12*0404 Allele. Arthritis Rheum 1999;42:569-573.

8. Bachmeyer C, Farge D, Gluckman E, et al. Raynaud's phenomenon and digital necrosis induced by interferon-alpha. Br J Dermatol 1996; 135: 481-483.

9. McMurray RW, Elbourne K. Hepatitis virus infection and autoimmunity. Semin Arthritis Rheum 1997;26:689-701.

10. Midgard R, Gronning M, Riise T, Kvale G, Nyland H. Multiple sclerosis and chronic inflammatory diseases: a case-control study. Acta Neurol Scand 1996;93:322-328.

11. Alsalameh S, Manger B, Kern P, Kalden J. New onset of rheumatoid arthritis during interferon b-1B treatment in a patient with multiple sclerosis: comment on the case report by Jabaily and Thompson [Letter]. Arthritis Rheum 1998;41:754.

12. Linden D. Severe Raynaud's phenomenon associated with interferon- $\beta$ treatment for multiple sclerosis [Letter]. Lancet 1998;352:878-879.

13. Sonnenblick M, Rosin A. Cardiotoxicity of interferon. Chest 1991;99:557-561.

14. Tada H, Saitoh S, Nakagawa Y, et al. Ischemic colitis during interferon-alpha treatment for chronic active hepatitis C. J Gastroenterol 1996;31: 582-584.

15. Purvin VA. Anterior ischemic optic neuropathy secondary to interferon-alpha. Arch Ophthalmol 1995;113:1041-1044.

16. Guyer DR, Tiedman J, Yannuzzi L, et al. Ineterferon-associated retinopathy. Arch Ophthalmol. 1993;111:350-356. 